IAC-07- 6.2.03

\title{
AN ANALYSIS OF THE ORBITAL DISTRIBUTION OF SOLID ROCKET MOTOR SLAG
}

\author{
Matthew F. Horstman \\ ERC Incorporated, USA \\ matt.horstman-1@nasa.gov \\ Mark Mulrooney \\ MEI, USA \\ mark-mulrooney-1@nasa.gov
}

\begin{abstract}
The contribution made by orbiting solid rocket motors (SRMs) to the orbital debris environment is both potentially significant and insufficiently studied. A combination of rocket motor design and the mechanisms of the combustion process can lead to the emission of sufficiently large and numerous by-products to warrant assessment of their contribution to the orbital debris environment. These particles are formed during SRM tail-off, or the termination of burn, by the rapid expansion, dissemination, and solidification of the molten $\mathrm{Al}_{2} \mathrm{O}_{3}$ slag pool accumulated during the main burn phase of SRMs utilizing immersion-type nozzles.

Though the usage of SRMs is low compared to the usage of liquid fueled motors, the propensity of SRMs to generate particles in the $100 \mu \mathrm{m}$ and larger size regime has caused concern regarding their contributing to the debris environment. Particle sizes as large as $1 \mathrm{~cm}$ have been witnessed in ground tests conducted under vacuum conditions and comparable sizes have been estimated via ground-based telescopic and in-situ observations of sub-orbital SRM tail-off events.

Using sub-orbital and post recovery observations, a simplistic number-size-velocity distribution of slag from on-orbit SRM firings was postulated. In this paper we have developed more elaborate distributions and emission scenarios and modeled the resultant orbital population and its time evolution by incorporating a historical database of SRM launches, propellant masses, and likely location and time of particulate deposition. From this analysis a more comprehensive understanding has been obtained of the role of SRM ejecta in the orbital debris environment, indicating that SRM slag is a significant component of the current and future population.
\end{abstract}




\section{Introduction}

Combustion of solid propellant, rather than the liquid fueld alternative, produces a rocket motor of high thrust and low specific impulse at relatively low comparitive cost. The thrust is produced via an oxidation reaction within a solid matrix consiting typically of a synthetic rubber polymer, metalic aluminum, and ammonium perchorate. The resulting by-product is primarily 10 to $100 \mu \mathrm{m}$ diameter dust particles that do not pose a significant orbital debris hazard. The preponderance of evidence indicates however that dust is not the only remnant left by an SRM burn - much larger particles have been observed exiting the rocket nozzles, specifically at tail-off. These particles are conjectured to result from explosive boilover of the molten Aluminum Oxide $\left(\mathrm{Al}_{2} \mathrm{O}_{3}\right)$ slag pool that accumulates around the immersion nozzles common to many SRMs. A portion of this molten slag is jettisoned out of the nozzle and into free space $[1,3]$.

Arising from post combustion processes at low internal chamber pressures, these particles do not experience the disruptive shearing effects that occur during the main burn and therefore are inherently larger $(\sim 1 \mathrm{~mm}-5 \mathrm{~cm})$ than combustion dust. Given that a $5 \mathrm{~mm}$ particle can penetrate the International Space Station in sensitive locations, and a $3 \mathrm{~mm}$ particle an astronaut's space suit, it is important to understand the size distribution and orbital behavior of these larger particles in order to predict the hazard posed both currently and in the future.

\section{Assumptions}

The mass distribution from SRM firings has been shown to be bi-modal [1]. While there is a significant population in the submillimeter size range, our analysis is focused on the large particle population due to the obvious danger it poses to existing and future orbital platforms. As laid out in a previous SRM study [2], the size range gleaned from in-situ observations and ground tests appears to be between $1 \mathrm{~mm}$ and $5 \mathrm{~cm}$.

There exists very little data on the exact amount of slag material that any given SRM expels. In order to remedy this much more work needs to be done in terms of sampling on-orbit motors, for example: high resolution
IR observations of orbital firings and vacuum ground testing.

In lieu of better data, this study establishes a Gaussian size distribution centered at $1 \mathrm{~cm}$ with a $1 \mathrm{~mm}-10 \mathrm{~cm}$ range. Here the distribution is a Gaussian,

$$
f(x, \mu, \sigma)=\frac{1}{\sigma \sqrt{2 \pi}} e^{-(x-\mu)^{2} /\left(2 \sigma^{2}\right)}
$$

with $\mu=1 \mathrm{~cm}$ and $\sigma^{2}=9.10 \mathrm{~cm}^{2}$.

Converting to log space (1) is transformed,

$$
f(s, 0, \tau)=\frac{1}{\log _{10} \sigma \sqrt{2 \pi}} e^{-s^{2} /\left(2 \tau^{2}\right)}
$$

where,

$$
s=\log _{10} x, \log _{10} \mu=0 \text {,and } \tau=\log _{10} \sigma=.48
$$

This relationship is expressed as a continuous function to describe the rate of production of particles versus diameter, represented by $p$, for a generic motor as a function of $s$.

$$
p(s)=\frac{1}{\sqrt{2 \pi / \tau}} e^{-s^{2} /\left(2 \tau^{2}\right)}
$$

Integrating and scaling for an individual object's slag contribution in kilograms,

$$
\frac{M}{k} \int_{\max _{-} x}^{\min { }_{x} x} p(s) d s=(\text { total\#objects })
$$

where $\mathrm{k}$ is the scaling factor for the assumed spherical blobs of slag. The constant $k$ is defined by,

$$
\begin{aligned}
& k=\frac{\rho \sqrt{0.5 \pi}}{6000 \tau} \int_{-1}^{1} 10^{3 s} e^{-s^{2} /\left(2 \tau^{2}\right)} d s \\
& =4.17 \times 10^{-3}(\mathrm{~kg} / \# \text { particles })
\end{aligned}
$$

and $\mathrm{M}$ is the mass of slag in $\mathrm{kg}$.

For each SRM launch, the assumption is that 0.04 to $0.65 \%$ of the fuel mass is ejected as slag into the final orbit of the payload, and all particles are ejected with a size distribution established by equation 4 .

In order to utilize these analytic functions in the computer environment simulation, they had to be transformed into a discrete function. Equation 4 was sampled into 20 
equal size bins in log space, for $-1 \leq s \leq 1$. As shown in Figure 1, the mass dependent size distribution is reduced to an algorithm suitable for simulating the environment.

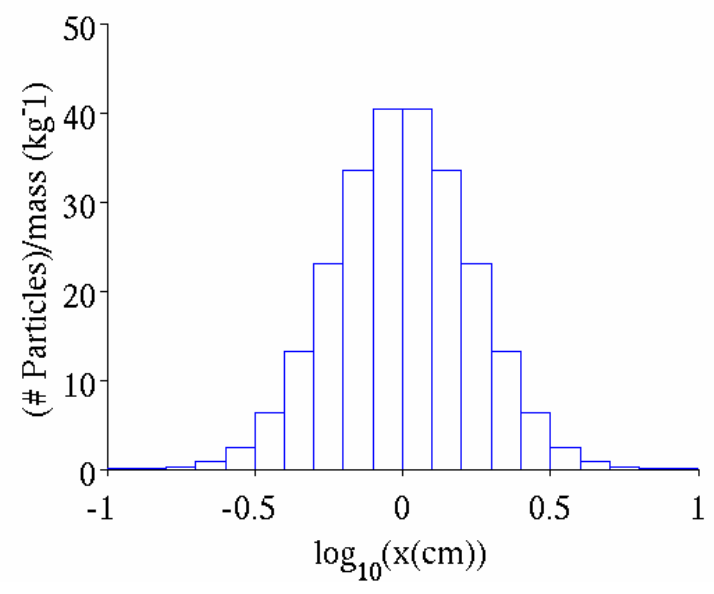

Figure 1. Discrete Size Distribution as a function of slag mass.

This analytic approach is demonstrated in Figure 2 as the distribution of particles from a Star 30 rocket motor with a total fuel mass of $464 \mathrm{~kg}$, which would have an estimated slag mass of $3.02 \mathrm{~kg}$.

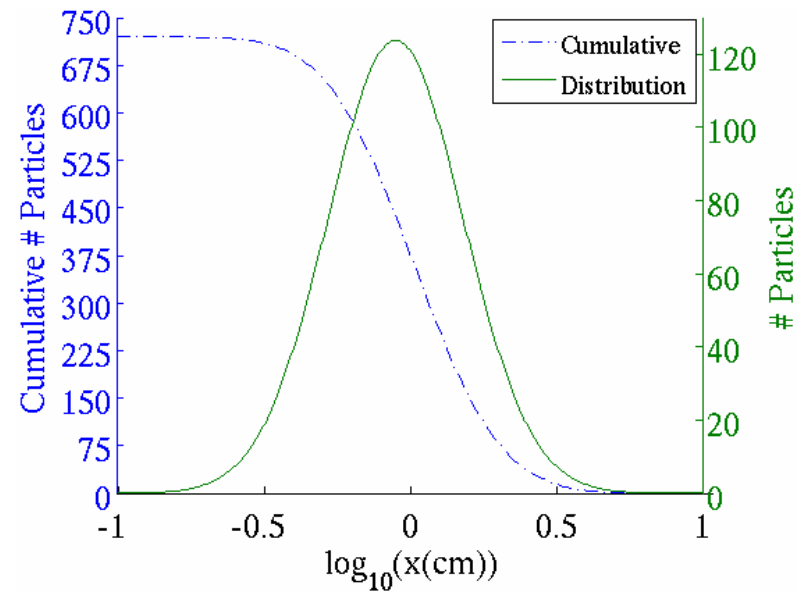

Figure 2. Particle distribution for a 3kg estimated slag ejection.

The pivotal assumption for slag ejecta is that the entire process occurs during the brief tail-off portion of the burn. Compared to the orbital period, SRM burns are of very short duration ( minutes) and tail-off is shorter still (typically seconds for the bulk ejection). Thus, it is a reasonable assumption that the burn occurs instantly when modeling the process. Slag ejection is thought to have very low velocity relative to the parent motor. Thus, for simplicity this term was assumed to be zero and the ejected slag is simply released upon tail-off. This means that the slag acquires the final orbital details of the payload delivered by the SRM.

\section{Launch Examples}

Examples of Low Earth Orbit (LEO), Geosynchronous Transfer Orbit (GTO) and Geosynchronous Earth Orbit (GEO) slag deposits were examined using the criteria presented here. An Orion 38 solid rocket motor was used for the LEO case, and a Star $30 \mathrm{C}$ was used in both the GTO and GEO cases.

The LEO launch used a Pegasus launch vehicle, containing an Orion 38 motor to achieve a final $840 \times 850 \mathrm{~km}$ orbit. This motor is contains $770 \mathrm{~kg}$ of fuel, which equates to 5 $\mathrm{kg}$ of slag, assuming that $0.65 \%$ of the total fuel mass is ejected as slag. Figure 3 depicts the launch and a $50 \mathrm{yr}$ projection of the particles to illustrate the residual slag.

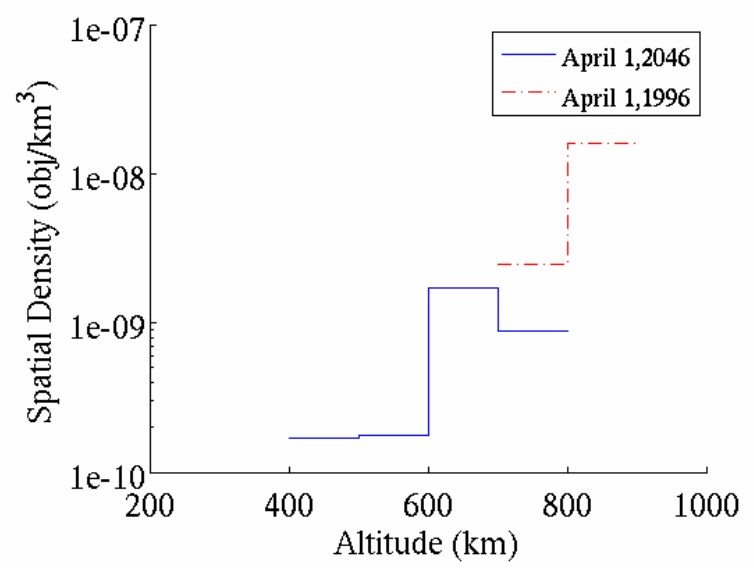

Figure 3. Spatial density of slag from a single LEO reference object.

For the GTO example a Star $30 \mathrm{C}$ was used to propel the payload into a $35,800 \mathrm{x}$ $220 \mathrm{~km}$ orbit. Reported to have a $586 \mathrm{~kg}$ fuel mass, the corresponding estimate of slag used was $3.8 \mathrm{~kg}$. Figure 4 illustrates how the environment is affected by the launch on March $29^{\text {th }}, 2002$ and shortly before the complete decay of particles less than a year later. 


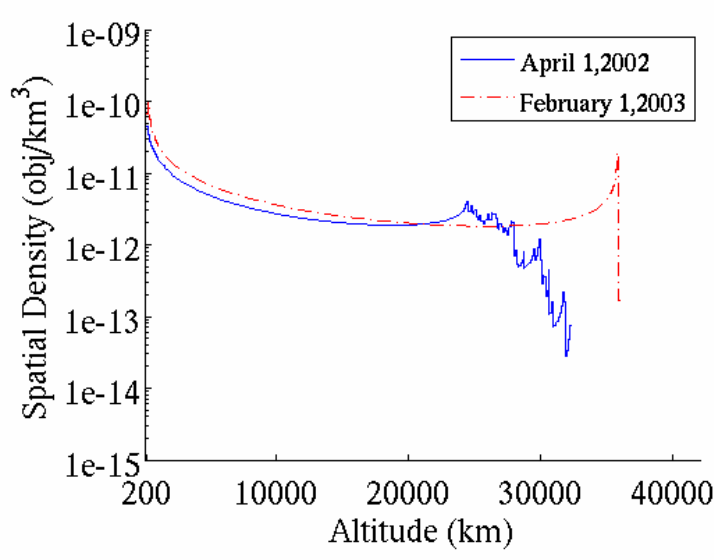

Figure 4. Spatial density of slag from a single GTO reference object.

Figure 5 shows the GEO case, which also uses a Star 30C motor. This SRM was used to circularize the payload into a $35,800 \times$ $35,800 \mathrm{~km}$ orbit in GEO, which is the most long-lived orbit studied in this paper. No particles decay from this orbit in the $50 \mathrm{yr}$ projection studied. In fact, nothing reenters for many thousands of years, thus the environment is most impacted by GEO circularizing burns, though the spatial density is much lower when compared to LEO.

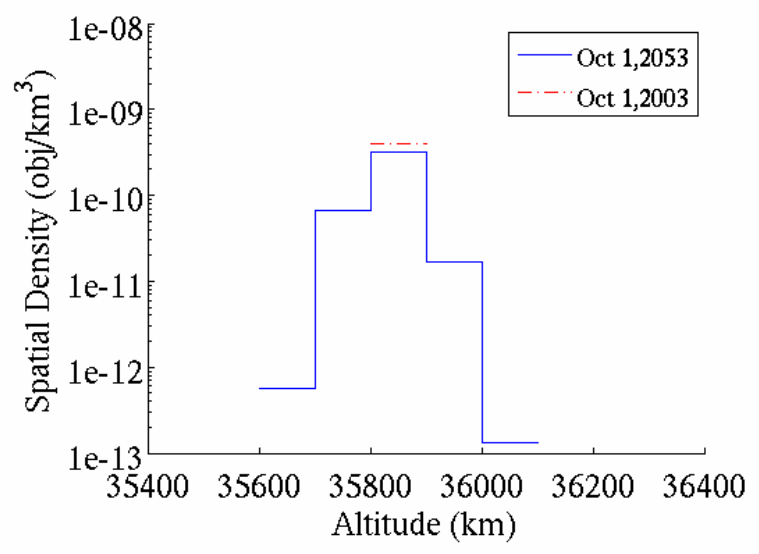

Figure 5. Spatial density of slag from a single GEO reference object.

As demonstrated in Figures 3 through 5, the decay rate of particles is varied resulting in varied orbit lifetimes for each slag particle.

The variation of $A / m$ seen in each slag cloud determines the rate at which each particle will decay from the environment. Figure 6 is a comparison between the Star $30 \mathrm{C}$ and Orion $38 \mathrm{~A} / \mathrm{m}$ distributions, which appear similar because they are the exact distribution scaled only by the mass of slag ejected. This is because the particle size distribution, (see Figure 2), relates diameter to mass and number of particles. $A / m$ is the ratio of the area to the mass of a slag particle, thus the $A / m$ distribution, (see Figure 6 ), and the size distribution are directly proportional to each other.

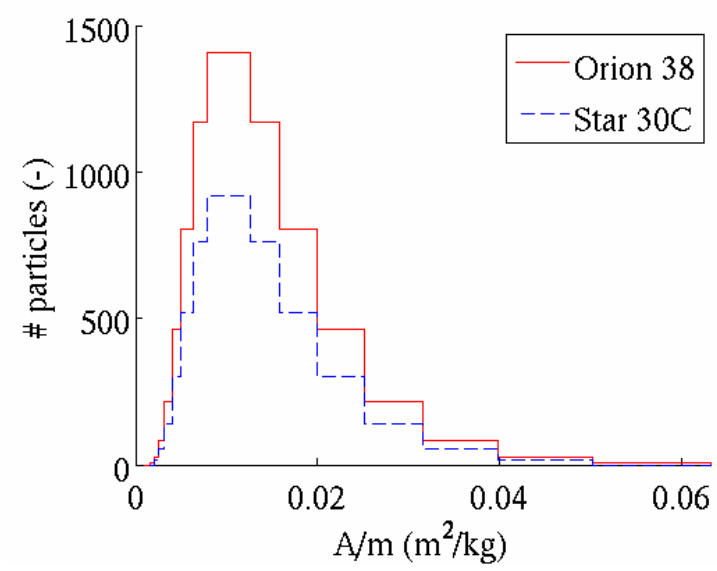

Figure 6. Histogram of $\mathrm{A} / \mathrm{m}$ for slag particles from both SRM motors.

After initial injection into the orbital environment, the cloud is distributed about the orbit achieved by the burn. As this cloud is propagated, the variation in $A / m$ causes portions of it to decay at different rates. Instead of a large block decaying at once, which would cause clumping in the spatial density, we see that this cloud disseminates into small clumps that populate each altitude as the group of similar $\mathrm{A} / \mathrm{m}$ objects decays.

\section{Historical SRM Launches and the Environment}

Accurately defining the SRM slag portion of the orbital environment in a comprehensive manner requires an accounting of each solid rocket motor launched, its orbit, the size of it associated particle cloud, and propagation of that cloud over time. We have initiated this broad survey to generate a detailed analysis of the contribution of SRM slag to the current and future environment.

Before the 1990s, SRM launches occurred at a rate of approximately 18 per year. It has since declined sharply to about 7 launches per year and that rate is expected to continue. Despite the decline, this still represents a significant amount of SRM slag injected into the orbital environment every year. Some common large solid rocket systems still in use include the Thiokol Star series motors, Pegasus, M-5, Start and Shavit rockets. Some are used to push payloads into semi-synchronous and GEO orbits. Others, such as the Pegasus, launch mostly into LEO. Slag ejection into the GEO 
realm is by far the most long-lived. The GTO and LEO payloads occupy the majority of SRM uses though, and slag resulting from these has a much shorter lifetime.

Now given the details of an individual cloud, the contribution to the orbital environment by multiple slag clouds over time is now examined. Using published details about each payload with an SRM, the launch traffic over an 8 year period was compiled along with the details of each burn, see Figure 7. This "Typical Launch Cycle", is drawn from all launches using known SRMs from January 1, 1996 to January 1, 2004. Additional historical launches will be added in subsequent papers.

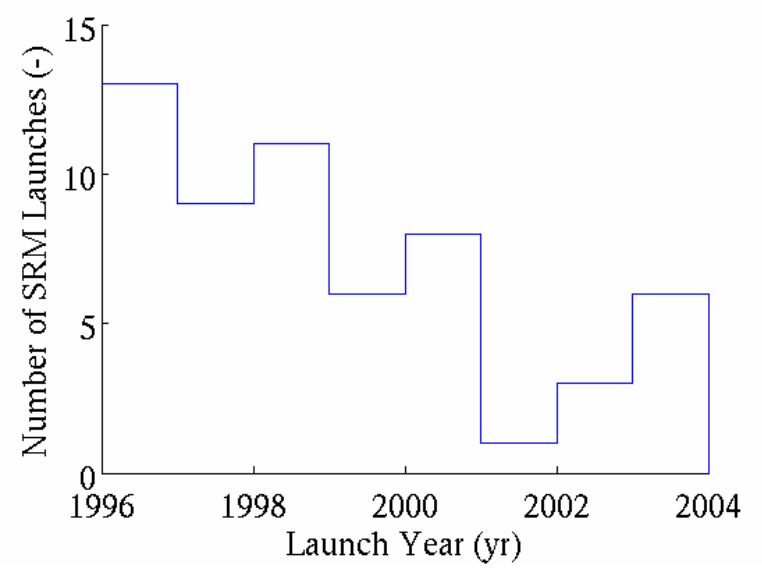

Figure 7. Reference Launch Traffic projection cycle by year for the first cycle only.

The launch and deposition rates naturally track each other, as shown in Figure 8. This represents how significant the launched reference cycle slag masses are.
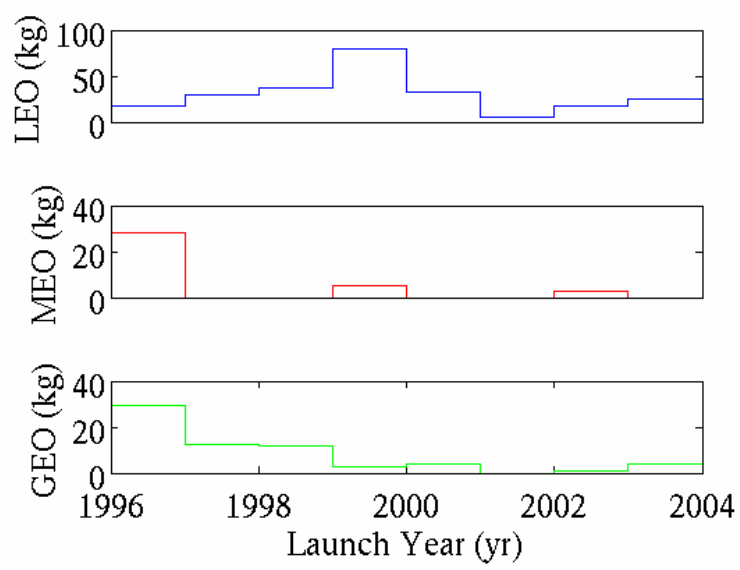

Figure 8. Reference Launch Slag Mass of projected cycle for the first cycle only.

In modeling the future this population was repeated every 8 years for a 50 year period. The spatial density from LEO to GEO in
January of 2006 and 2057 is shown in Figure 9.

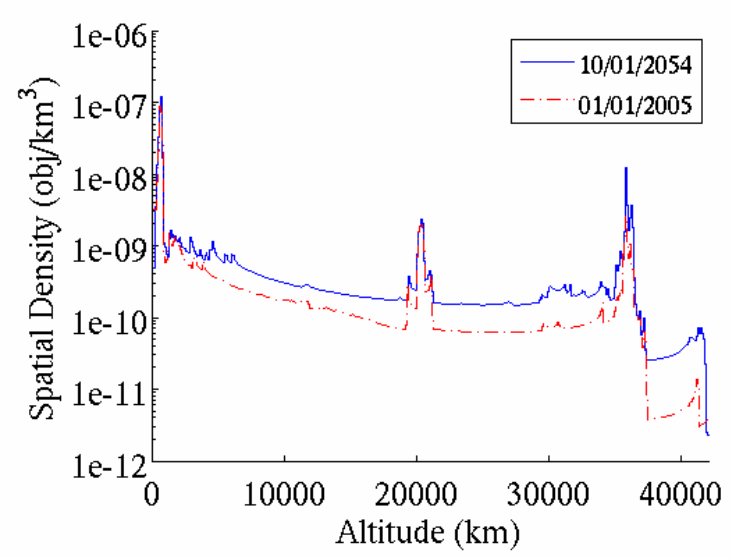

Figure 9. Spatial Density for 8 year Launch Cycle (2005) and the cumulative population after 8 total cvcles (2054).

Projecting this cycle into the future, the chart illustrates how the 8 year reference cycle adds up over time in much of the high altitude orbits, and after 7 cycles appears to be merely a scaled up density when compared to the first cycle in 2005. To underscore its importance, Figure 10 shows the significance of the slag contribution relative to the total environment in LEO, as measure by the Haystack radar. [9]

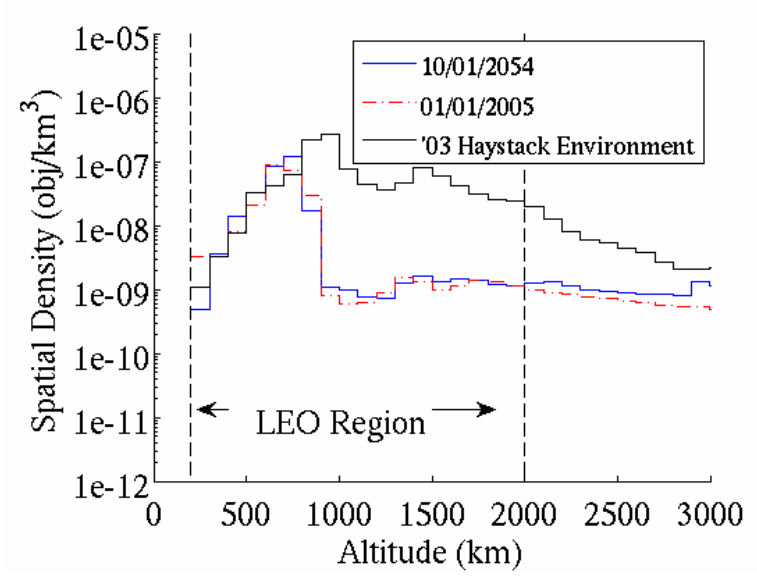

Figure 10. LEO Spatial Density for 8 year Launch Cycle and the cumulative population after 8 total cycles $(x>1 \mathrm{~cm}$ ).

The dynamic nature of these small particles is illustrated by Figure 10. For altitudes below $2000 \mathrm{~km}$, the residual debris population from past cycles is very low. When the first and last cycles are compared against each other, Figure 9 illustrates how the population above $2000 \mathrm{~km}$ has a much longer lifetime than the LEO population. The 
measured debris population was sampled by the Haystack Radar in 2003, and charted along with these populations for comparison. The SRM environment estimation appears to be skewed towards overpopulation in the regions below $800 \mathrm{~km}$ after only the first cycle. This could be due in part to an overestimate in the percentage of fuel converted to slag.

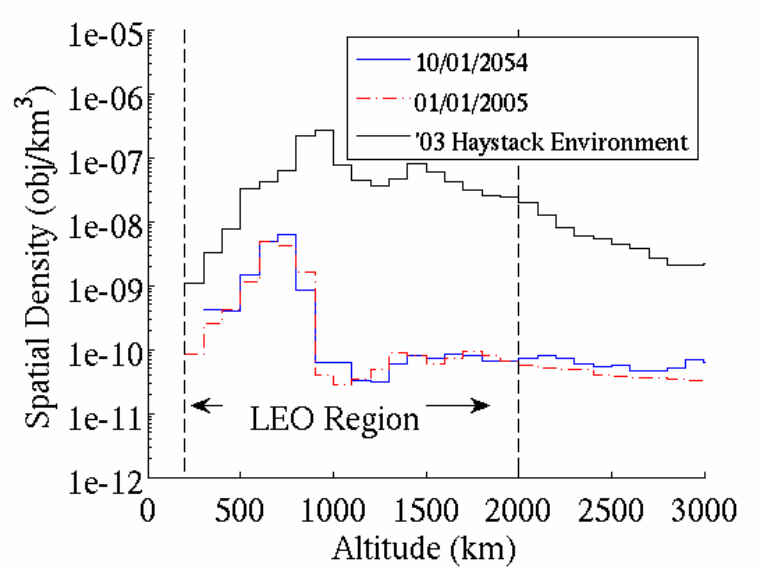

Figure 11. Spatial Density for 8 year Launch Cycle (2005) and the cumulative population after 8 total cycles (2054) for LEO region ( $x>1 \mathrm{~cm}$ ), using the $0.04 \%$ fuel to slag mass estimate.

Reducing the mass ejected as slag produced a LEO population that was lower, and thus fit better to the radar sampled environment. By reducing this mass, the number of particles ejected were reduced at all altitudes. Decreasing the slag mass from the $0.65 \%$ of the fuel mass to the minimum estimate of $0.04 \%$, as shown in Figure 11 , shows a bracketed region from which the approximate slag population can be drawn. Thie range is based upon the assumption that slag ejection is established as $[0.04 \%, 0.65 \%]$. [2] As shown in Figure 12, the overall environment reflects the reduction in SRM slag from LEO to GEO altitudes.

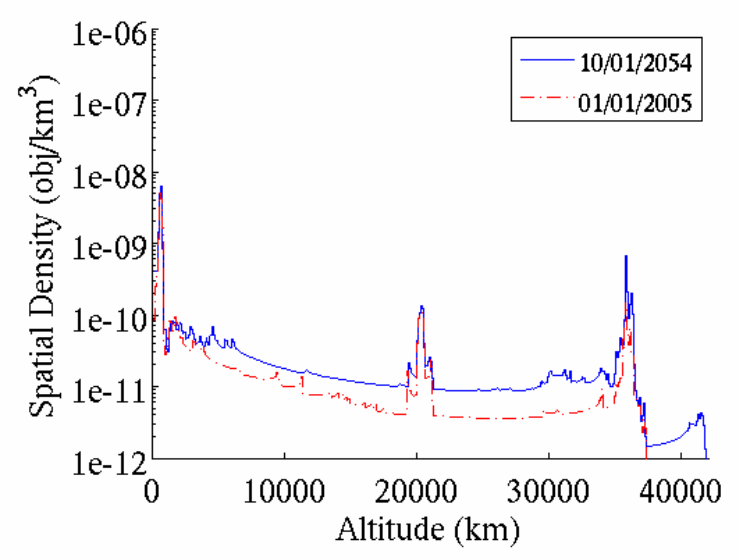

Figure 12. Spatial Density for 8 year Launch Cycle (2005) and the cumulative population after 8 total cycles (2054) $(x>1 \mathrm{~cm}$ ), using the $0.04 \%$ fuel to slag mass estimate.

\section{Conclusion}

Orbiting payloads utilizing solid rocket motors for propulsion contribute directly to the degradation of the orbiting environment via the ejection of slag. Although the use of solid rocket motors has steadily decreased, past emissions will continue to influence the environment indefinitely, as will the few remaining annual launches. To better understand the extent and nature of SRM effluents, a comprehensive study of the particulates ejected during either groundbased vacuum or on-orbit firings should be considered. Gathering and sorting the $1 \mathrm{~mm}$ and greater ejecta from an SRM fired in vacuum may yield a higher fidelity model than the simple Gaussian assumed here. In the meantime, as debris modeling continues to evolve, the incorporation of slag into the overall debris model is helping us gain a more complete understanding of the risks involved in using our orbital resources. Going forward we intend to explore alternate size and velocity distributions and encompass more historical launches in our models.

\section{References}

1. Salita, M., "Deficiencies and Requirements in Modelling of Slag Generation in Solid Rocket Motors", Journal of Propulsion and Power, Vol 11-1, 1995.

2. Mulrooney, M., An Assessment of the Role of Solid Rocket Motors in The Generation of Orbital Debris, NASA/TP-2007-213738, 2007. 3. Johnson, N., Potter, A., "Orbital Debris from Solid Rocket Motor Slag", IAF-96V.6.04, 1996.

4. NRC, Protecting the Space Shuttle from Meteoroids and Orbital Debris, National Academy Press, 1997.

5. Mulrooney, M., "An Assessment of the Role of Solid Rocket Motors in the Generation of Orbital Debris", Orbital Debris Quarterly News, volume 8, issue 4.

6. McDowell, J., "Kick In The Apogee: 40 Years of Upper Stage Applications For Solid Rocket Motors, 1957-1997", AIAA 97-3133, July, 1997. 
7. Wade, M., Encyclopedia Astronautica, http://www.astronautix.com (access April 2006)

8. Isakowitz, S., International Reference Guide to Space Launch Systems, AIAA, Reston, VA, 2004.

9. Xu, Yu-lin, "Statistical Inference In Modeling The Orbital Debris Environment”, B6.2.03, IAC 2006. 